\title{
KEPLER-7b: A TRANSITING PLANET WITH UNUSUALLY LOW DENSITY*
}

\author{
David W. Latham ${ }^{1}$, William J. Borucki ${ }^{2}$, David G. Koch $^{2}$, Timothy M. Brown ${ }^{3}$, Lars A. Buchhave ${ }^{1,4}$, Gibor Basri ${ }^{5}$, \\ Natalie M. Batalha ${ }^{6}$, Douglas A. Caldwell ${ }^{7}$, William D. Cochran ${ }^{8}$, Edward W. Dunham ${ }^{9}$, Gabor Fúrész $^{1}$, \\ Thomas N. Gautier III ${ }^{10}$, John C. Geary ${ }^{1}$, Ronald L. Gilliland ${ }^{11}$, Steve B. Howell ${ }^{12}$, Jon M. Jenkins ${ }^{7}$, Jack J. Lissauer ${ }^{2}$, \\ Geoffrey W. Marcy ${ }^{4}$, David G. Monet $^{13}$, Jason F. Rowe ${ }^{2,14}$, and Dimitar D. Sasselov ${ }^{1}$ \\ ${ }^{1}$ Harvard-Smithsonian Center for Astrophysics, 60 Garden Street, Cambridge, MA 02138, USA \\ ${ }^{2}$ NASA Ames Research Center, Moffett Field, CA 94035, USA \\ ${ }^{3}$ Las Cumbres Observatory Global Telescope, Goleta, CA 93117, USA \\ ${ }^{4}$ Niels Bohr Institute, Copenhagen University, DK-2100 Copenhagen, Denmark \\ ${ }^{5}$ University of California-Berkeley, Berkeley, CA 94720, USA \\ ${ }^{6}$ San Jose State University, San Jose, CA 95192, USA \\ ${ }^{7}$ SETI Institute, Mountain View, CA 94043, USA \\ ${ }^{8}$ University of Texas, Austin, TX 78712, USA \\ ${ }^{9}$ Lowell Observatory, Flagstaff, AZ 86001, USA \\ 10 Jet Propulsion Laboratory/California Institute of Technology, Pasadena, CA 91109, USA \\ ${ }^{11}$ Space Telescope Science Institute, Baltimore, MD 21218, USA \\ ${ }^{12}$ National Optical Astronomy Observatory, Tucson, AZ 85719, USA \\ ${ }^{13}$ US Naval Observatory, Flagstaff Station, Flagstaff, AZ 86001, USA \\ Received 2009 November 16; accepted 2010 January 5; published 2010 March 30
}

\begin{abstract}
We report on the discovery and confirmation of Kepler-7b, a transiting planet with unusually low density. The mass is less than half that of Jupiter, $M_{\mathrm{P}}=0.43 M_{\mathrm{J}}$, but the radius is $50 \%$ larger, $R_{\mathrm{P}}=1.48 R_{\mathrm{J}}$. The resulting density, $\rho_{\mathrm{P}}=0.17 \mathrm{~g} \mathrm{~cm}^{-3}$, is the second lowest reported so far for an extrasolar planet. The orbital period is fairly long, $P=4.886$ days, and the host star is not much hotter than the Sun, $T_{\mathrm{eff}}=6000 \mathrm{~K}$. However, it is more massive and considerably larger than the Sun, $M_{\star}=1.35 M_{\odot}$ and $R_{\star}=1.84 R_{\odot}$, and must be near the end of its life on the main sequence.
\end{abstract}

Key words: planetary systems - stars: individual (Kepler-7, KIC 5780885, 2MASS 19141956+4105233) techniques: spectroscopic

Online-only material: color figures

\section{INTRODUCTION}

The final test of the Kepler photometer at the end of commissioning was a run of 9.7 continuous days in science mode, to evaluate the noise performance of the instrument. The Kepler Input Catalog (KIC) was used to select 50,000 isolated targets, all with magnitudes brighter than 13.8 in the Kepler passband, and with no nearby companions that would contaminate the photometry. The preliminary light curves from this test run were inspected by team members with great excitement, and a few dozen obvious planet candidates were quickly identified and passed on to the team responsible for ground-based followup observations. Kepler-7 was observed but was not identified among the sample of initial candidates.

After a gap of 1.3 days, normal science observations began for a full list of more than 150,000 planet-search targets and continued for 33.5 days until interrupted on 2009 June 15, followed by a data download and roll of the spacecraft to the summer orientation. By the middle of July the preliminary light curves were available for inspection, and dozens of additional candidates were identified and passed on to the follow-up team. This time Kepler-7 was included. Along with the other candidates, Kepler-7 was scrutinized for evidence of astrophysical false positives involving eclipsing binaries. It

\footnotetext{
* Based in part on observations obtained at the W. M. Keck Observatory, which is operated by the University of California and the California Institute of Technology.

${ }^{14}$ NASA Postdoctoral Program Fellow.
}

survived this stage of the follow-up and was then observed spectroscopically for very precise radial velocities using the FIber-fed Echelle Spectrograph (FIES) on the Nordic Optical Telescope (NOT) during a ten night run in early October. These observations yielded a spectroscopic orbit that confirmed that an unseen companion with a planetary mass was responsible for the dips in the light curve observed by Kepler.

The KIC used ground-based multi-band photometry to assign an effective temperature and surface gravity of $T_{\text {eff }}=5944 \mathrm{~K}$ and $\log g=4.27$ (cgs) to Kepler-7, corresponding to a late-F or early-G dwarf. Stellar gravities in this part of the H-R diagram are notoriously difficult to determine from photometry alone, and one of the conclusions of this Letter is that the star is near the end of its main-sequence lifetime, with a radius that has expanded to $R_{\star}=1.843_{-0.066}^{+0.048} R_{\odot}$ and a surface gravity that has weakened to $\log g=4.030_{-0.019}^{+0.018}$ (cgs). In turn, this implies an inflated radius for the planet, resulting in an unusually low density of $\rho_{\mathrm{P}}=0.17 \mathrm{~g} \mathrm{~cm}^{-3}$. This conclusion is hard to avoid, because the relatively long duration of the transit, more than $5 \mathrm{hr}$ from first to last contact, demands a low density and expanded radius for the star.

\section{KEPLER PHOTOMETRY}

The light curve for Kepler-7 $(=$ KIC 5780885, $\alpha=$ $19^{\mathrm{h}} 14^{\mathrm{m}} 19.56, \delta=+41^{\circ} 05^{\prime} 23^{\prime \prime} \cdot 3, \mathrm{~J} 2000$, KIC $\left.r=12.815 \mathrm{mag}\right)$ is plotted in Figure 1. The numerical data are available electronically from the Multi Mission Archive at the Space Telescope Science Institute (MAST) High Level Science Products (HLSP) 

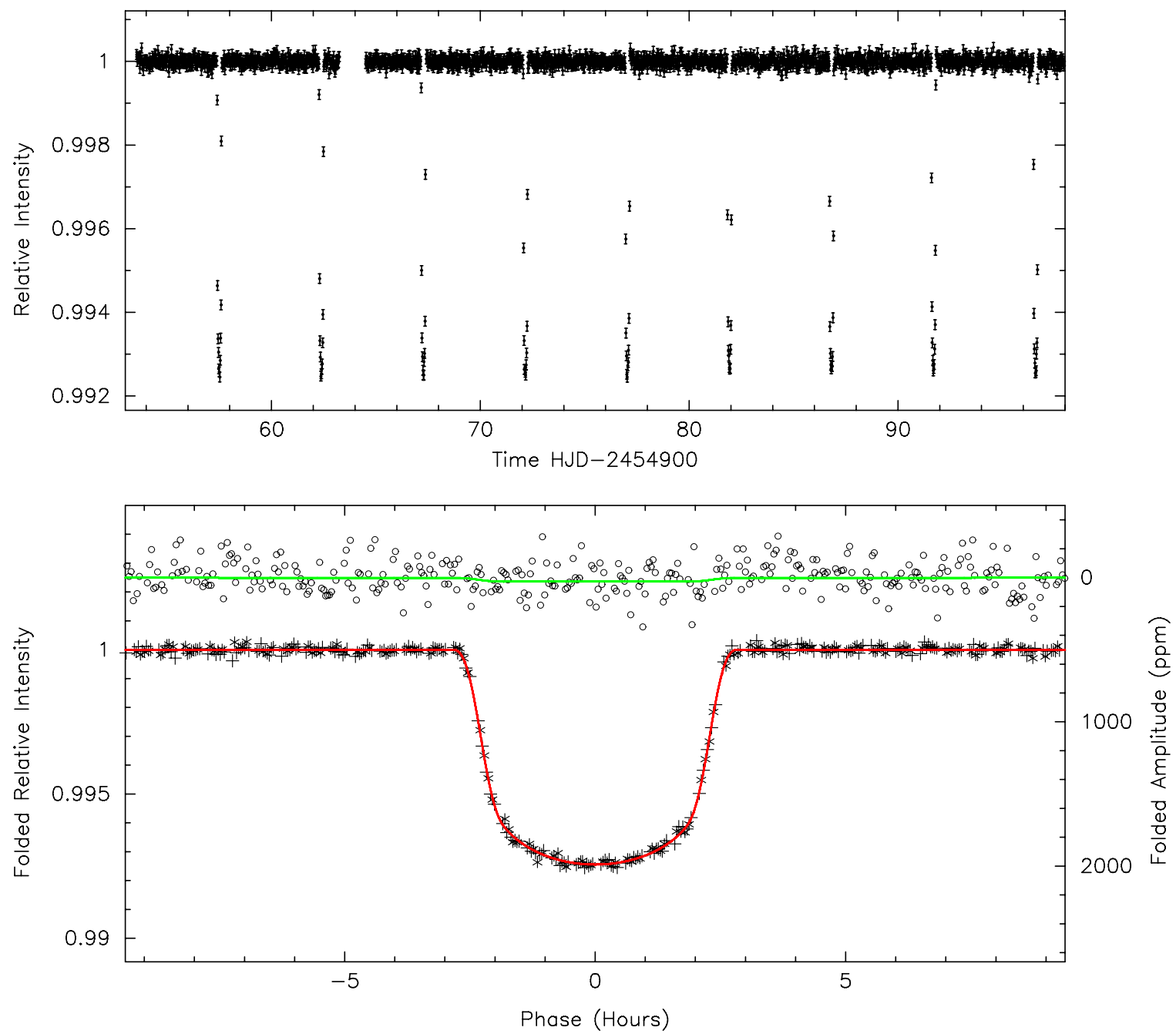

Figure 1. Detrended light curve for Kepler-7. The time series for the entire data set is plotted in the upper panel. The lower panel shows the photometry folded by the period $P=4.885525$ days. The model fit to the primary transit is plotted in red, and our attempt to fit a corresponding secondary eclipse for a circular orbit is shown in green with an expanded and offset scale.

(A color version of this figure is available in the online journal.)

Web site. ${ }^{15}$ Only a modest amount of detrending has been applied (Koch et al. 2010) to this time series of long cadence data (29.4 minute accumulations). There is no evidence for any systematic difference between alternating events, which are plotted with + and $\times$ symbols, supporting the interpretation that all the events are primary transits. Indeed, there $i$ s weak evidence for a secondary eclipse centered at phase 0.5 , as would be expected for a circular orbit, but the significance is only about $2.4 \sigma$. If this detection is real, it is not inconsistent with the thermal emission expected from the planet for reasonable assumptions (Koch et al. 2010).

\section{FOLLOW-UP OBSERVATIONS}

As described in more detail by Gautier et al. (2010), the initial follow-up observations of Kepler planet candidates involved reconnaissance spectroscopy to look for evidence of a stellar companion or a nearby eclipsing binary responsible for the observed transits. However, the follow-up team soon learned that the astrometry derived from the Kepler images themselves, when combined with high-resolution images of the target neighborhood, could provide a very powerful tool for identifying

\footnotetext{
15 http://archive.stsci.edu/prepds/kepler_hlsp
}

background eclipsing binaries blended with and contaminating the target images (Batalha et al. 2010; Monet et al. 2010). The astrometry of Kepler-7 indicated a very slight image centroid shift during transits of +0.1 millipixels in its $C C D$ row direction only.

The only star listed in the KIC that is closer than $30^{\prime \prime}$ to Kepler-7 and that can contribute significant light to the Kepler-7 photometry is KIC 5780899, which is 4.4 mag fainter and lies at a separation of 15".5. KIC 5780899 cannot be the source of the observed dips, because that would induce centroid shifts of about 25 millipixels. If KIC 5780899 is constant and Kepler-7 is the source of the transits, the predicted shifts are in the right direction and have an amplitude of roughly 0.1 millipixels if a quarter of KIC 5780899's light leaks into the Kepler-7 aperture. Thus, KIC 5780899 provides a satisfactory explanation for the observed shifts.

To check for very close companions, a speckle observation of Kepler-7 was obtained by S. Howell with the WIYN $3.5 \mathrm{~m}$ telescope on Kitt Peak. It showed no companions in a $2^{\prime \prime}$ box centered on Kepler-7. Subsequently, images obtained by H. Isaacson with the High Resolution Echelle Spectrometer (HIRES) guider on Keck 1, and independently by G. Mandushev with the $1.8 \mathrm{~m}$ Perkins telescope and PRISM camera at the 
Table 1

Relative Radial Velocity Measurements of Kepler-7

\begin{tabular}{ccccrr}
\hline \hline $\begin{array}{c}\text { HJD } \\
\text { (days) }\end{array}$ & $\begin{array}{c}\text { Phase } \\
(\text { cycles })\end{array}$ & $\begin{array}{c}\text { RV } \\
\left(\mathrm{m} \mathrm{s}^{-1}\right)\end{array}$ & $\begin{array}{c}\sigma_{\mathrm{RV}} \\
\left(\mathrm{m} \mathrm{s}^{-1}\right)\end{array}$ & $\begin{array}{c}\mathrm{BS} \\
\left(\mathrm{m} \mathrm{s}^{-1}\right)\end{array}$ & $\begin{array}{r}\sigma_{\mathrm{BS}} \\
\left(\mathrm{m} \mathrm{s}^{-1}\right)\end{array}$ \\
\hline 2455107.37937 & 28.677 & +43.7 & \pm 6.8 & +19.9 & \pm 7.3 \\
2455108.36845 & 28.879 & +32.7 & \pm 7.1 & +1.5 & \pm 5.4 \\
2455110.50735 & 29.317 & -34.2 & \pm 9.8 & +4.8 & \pm 17.9 \\
2455111.40251 & 29.500 & -11.5 & \pm 6.7 & -4.0 & \pm 7.2 \\
2455112.41378 & 29.707 & +33.2 & \pm 8.2 & -4.6 & \pm 5.4 \\
2455113.40824 & 29.911 & +27.9 & \pm 6.1 & -12.0 & \pm 8.2 \\
2455114.44632 & 30.123 & -31.1 & \pm 8.1 & -5.5 & \pm 8.9 \\
2455115.44411 & 30.328 & -29.2 & \pm 10.7 & -14.8 & \pm 8.9 \\
2455116.37077 & 30.517 & -0.1 & \pm 9.4 & +13.8 & \pm 10.6 \\
\hline
\end{tabular}

Lowell Observatory and by N. Baliber with the LCOGT Faulkes Telescope North on Haleakala, Maui, all detected a companion at a separation of 1".8 (just outside the WIYN speckle window) and about 4.4 mag fainter in the red. This companion cannot be the source of the observed centroid shifts. If it is the source of the dips in the light curve, the centroid shifts would have to be larger than 1 millipixel, and in the wrong direction. If it is constant, the shifts would be much too small to detect. However, this companion does dilute the photometry of Kepler-7 with a contribution of about $2.1 \%$. Adding in a quarter of the light from the more distant companion gives a total dilution of about $2.5 \pm 0.4 \%$. This dilution has been included in the analysis of the light curve.

Reconnaissance spectra obtained by M. Endl and W. Cochran with the coudé echelle spectrograph on the $2.7 \mathrm{~m}$ Harlan J. Smith Telescope at the McDonald Observatory showed that there was no significant velocity variation at the level of $1 \mathrm{~km} \mathrm{~s}^{-1}$, and therefore that an orbiting stellar companion could not be responsible for the observed transits. Furthermore, there was no sign of a composite spectrum or contamination by the spectrum of an eclipsing binary. The McDonald spectra were classified by L. Buchhave by finding the best match between the observed spectra and a library of synthetic spectra calculated by J. Laird for an extensive grid of stellar models (Kurucz 1992) using a line list developed by J. Morse. This yielded $T_{\text {eff }}=6000 \pm 125 \mathrm{~K}$, $\log g=4.0 \pm 0.2(\mathrm{cgs})$, and $v \sin i=4 \mathrm{~km} \mathrm{~s}^{-1}$, very close to the final values reported in Table 2 .

\section{FIES SPECTROSCOPY}

The FIES on the $2.5 \mathrm{~m}$ NOT at La Palma was not originally designed with very precise radial velocities in mind. In particular, the fiber feed does not incorporate a scrambler, there is no attempt to control the atmospheric pressure (e.g., by housing the optics in a vacuum enclosure), and there is no correction of the images for atmospheric dispersion. However, the spectrograph does reside in its own well-insulated room with active control of the temperature to a few hundredths kelvin, with the result that the optics are quite stable. Furthermore, FIES has good throughput, partly because the seeing is often excellent at the NOT site, and an automatic guider keeps the image well centered on a fiber 1".3 in diameter. These advantages encouraged us to develop specialized observing procedures and a new data reduction pipeline with the goal of measuring radial velocities to better than $10 \mathrm{~m} \mathrm{~s}^{-1}$ for the relatively faint planet candidates identified by Kepler.

To establish a wavelength calibration that tracks slow drifts during a long exposure, we adopted the strategy of obtaining strong exposures of a thorium-argon hollow cathode lamp

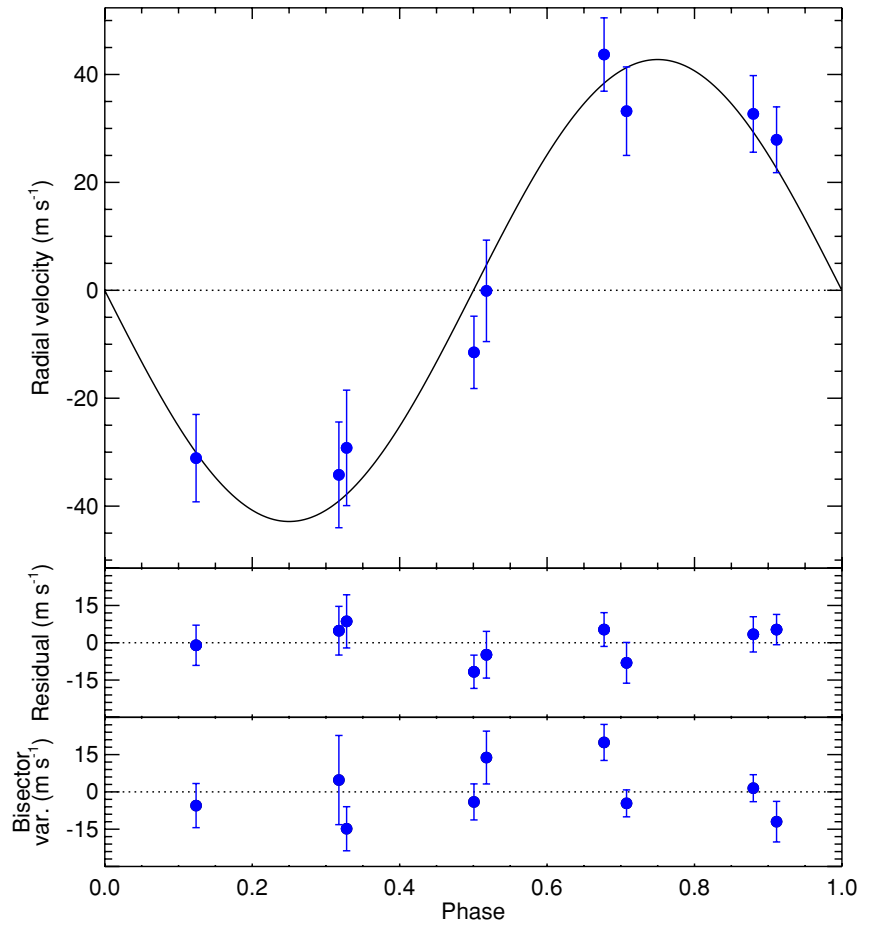

Figure 2. (a) Orbital solution for Kepler-7. The observed radial velocities obtained with FIES on the NOT are plotted together with the velocity curve for a circular orbit with the period and time of transit fixed by the photometric ephemeris. The $\gamma$ velocity has been subtracted from the relative velocities here and in Table 1, and thus the center-of-mass velocity for the orbital solution is 0 by definition. (b) Velocity residuals from the orbital solution. The rms of the velocity residuals is $7.4 \mathrm{~m} \mathrm{~s}^{-1}$. (c) Variation in the bisector spans for the nine FIES spectra. The mean value has been subtracted.

(A color version of this figure is available in the online journal.)

through the science fiber immediately before and after each science exposure. Long science exposures are divided into three or more sub-exposures, to allow detection of and correction for radiation events. Contamination by scattered moonlight can be a serious problem for very precise velocities of faint targets. FIES does not yet have a separate fiber for monitoring the sky brightness, so care is needed to avoid the Moon, especially if there are thin clouds.

A new reduction and analysis pipeline optimized for measuring precise radial velocities was developed by L. Buchhave. After extraction of intensity- and wavelength-calibrated spectra, relative velocities are derived for each echelle order by cross correlation against a combined template created by shifting all the observed spectra of the same star to a common velocity scale and co-adding them. The final velocity for each observation is the mean of the results for the individual orders, weighted by the number of detected photons but not by the velocity information content. Orders with very low signal levels and orders contaminated by telluric lines are not used. The internal error of the mean is estimated from the scatter over the orders.

We observed Kepler-7 with FIES for an hour on each of the 10 consecutive nights in 2009 October. On every night we observed a standard star, HD 182488, soon before Kepler-7 and also soon after on half the nights. HD 182488 is conveniently located close to the Kepler field of view and is known from HIRES observations over several years to be stable to better than $3 \mathrm{~m} \mathrm{~s}^{-1}$, and thus was adopted as the primary velocity standard by the follow-up team. Our 15 velocities for HD 182488 show an rms of $7 \mathrm{~m} \mathrm{~s}^{-1}$, with a slow drift pattern with an amplitude of several $\mathrm{m} \mathrm{s}^{-1}$. Therefore, we interpolated a correction to our 
Table 2

System Parameters for Kepler-7

\begin{tabular}{|c|c|c|}
\hline Parameter & Value & Notes \\
\hline \multicolumn{3}{|l|}{ Transit and orbital parameters } \\
\hline Orbital period $P(\mathrm{~d})$ & $4.885525 \pm 0.000040$ & $\mathrm{~A}$ \\
\hline Midtransit time $E$ (HJD) & $2454967.27571 \pm 0.00014$ & A \\
\hline Scaled semimajor axis $a / R_{\star}$ & $7.22_{-0.13}^{+0.16}$ & A \\
\hline Scaled planet radius $R_{\mathrm{P}} / R_{\star}$ & $0.08241_{-0.00043}^{+0.00030}$ & A \\
\hline Impact parameter $b \equiv a \cos i / R_{\star}$ & $0.445_{-0.044}^{+0.032}$ & A \\
\hline Orbital inclination $i(\mathrm{deg})$ & $86.5 \pm 0.4$ & A \\
\hline Orbital semi-amplitude $K\left(\mathrm{~m} \mathrm{~s}^{-1}\right)$ & $42.9 \pm 3.5$ & A,B \\
\hline Orbital eccentricity $e$ & 0 (adopted) & $\mathrm{A}, \mathrm{B}$ \\
\hline Center-of-mass velocity $\gamma\left(\mathrm{m} \mathrm{s}^{-1}\right)$ & 0 & $\mathrm{~A}, \mathrm{~B}$ \\
\hline \multicolumn{3}{|l|}{ Observed stellar parameters } \\
\hline Effective temperature $T_{\text {eff }}(\mathrm{K})$ & $5933 \pm 44$ & $\mathrm{C}$ \\
\hline Spectroscopic gravity $\log g$ (cgs) & $3.98 \pm 0.10$ & $\mathrm{C}$ \\
\hline Metallicity $[\mathrm{Fe} / \mathrm{H}]$ & $+0.11 \pm 0.03$ & $\mathrm{C}$ \\
\hline Projected rotation $v \sin i\left(\mathrm{~km} \mathrm{~s}^{-1}\right)$ & $4.2 \pm 0.5$ & $\mathrm{C}$ \\
\hline Mean radial velocity $\left(\mathrm{km} \mathrm{s}^{-1}\right)$ & $+0.40 \pm 0.10$ & $\mathrm{~B}$ \\
\hline \multicolumn{3}{|l|}{ Derived stellar parameters } \\
\hline Mass $M_{\star}\left(M_{\odot}\right)$ & $1.347_{-0.054}^{+0.072}$ & C,D \\
\hline Radius $R_{\star}\left(R_{\odot}\right)$ & $1.843_{-0.066}^{+0.048}$ & C,D \\
\hline Surface gravity $\log g_{\star}(\mathrm{cgs})$ & $4.030_{-0.019}^{+0.018}$ & C,D \\
\hline Luminosity $L_{\star}\left(L_{\odot}\right)$ & $4.15_{-0.54}^{+0.63}$ & C,D \\
\hline Age (Gyr) & $3.5 \pm 1.0$ & C,D \\
\hline \multicolumn{3}{|l|}{ Planetary parameters } \\
\hline Mass $M_{\mathrm{P}}\left(M_{\mathrm{J}}\right)$ & $0.433_{-0.041}^{+0.040}$ & $\mathrm{~A}, \mathrm{~B}, \mathrm{C}, \mathrm{D}$ \\
\hline Radius $R_{\mathrm{P}}\left(R_{\mathrm{J}}\right.$, equatorial $)$ & $1.478_{-0.051}^{+0.050}$ & A,B,C,D \\
\hline Density $\rho_{\mathrm{P}}\left(\mathrm{g} \mathrm{cm}^{-3}\right)$ & $0.166_{-0.020}^{+0.019}$ & $\mathrm{~A}, \mathrm{~B}, \mathrm{C}, \mathrm{D}$ \\
\hline Surface gravity $\log g_{\mathrm{P}}(\mathrm{cgs})$ & $2.691_{-0.045}^{+0.038}$ & $\mathrm{~A}, \mathrm{~B}, \mathrm{C}, \mathrm{D}$ \\
\hline Orbital semimajor axis $a(\mathrm{AU})$ & $0.06224_{-0.00084}^{+0.00109}$ & E \\
\hline Equilibrium temperature $T_{\mathrm{eq}}(\mathrm{K})$ & $1540 \pm 200$ & $\mathrm{~F}$ \\
\hline
\end{tabular}

Notes.

A: based on the photometry.

$\mathrm{B}$ : based on the radial velocities.

C: based on a MOOG analysis of the FIES spectra.

D: based on the Yale-Yonsei stellar evolution tracks.

E: based on Newton's version of Kepler's third law and total mass.

$\mathrm{F}$ : assumes Bond albedo $=0.1$ and complete re-distribution.

velocity zero point for each observation of Kepler-7 by assuming that HD 182488 should not vary. One of the 10 observations was obtained through clouds and clearly showed a distortion of the correlation peak due to contamination by scattered moonlight for several of the blue orders. This observation was rejected. The results for the other nine observations are reported in Table 1, including the variations in the line bisectors and errors.

We fit a circular orbit to the nine velocities reported in Table 1, adopting the photometric ephemeris, which leaves the orbital semi-amplitude, $K$, and center-of-mass velocity, $\gamma$, as the only free parameters. A plot of this orbital solution is shown in Figure 2, together with the velocity residuals and the line bisector variations. There is no evidence of a correlation between the velocities and the bisectors, which supports the interpretation that the velocity variations are due to a planetary companion. The orbital parameters are listed in Table 2. Allowing the eccentricity to be a free parameter reduced the velocity residuals by only a small amount and yielded an eccentricity that was not significantly different from circular. A solution for a circular orbit using the velocities uncorrected for the drifts exhibited by the standard star gave similar velocity residuals, but a smaller value of $K$ by $7.6 \mathrm{~m} \mathrm{~s}^{-1}$, corresponding to an $18 \%$ smaller mass.

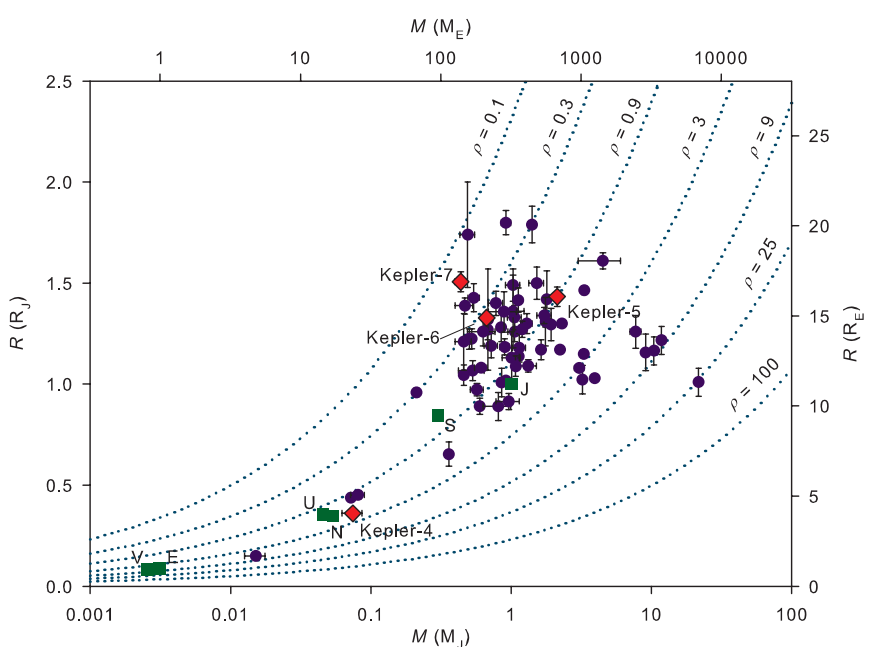

Figure 3. Mass/radius diagram for all the transiting planets with known parameters as of 2009 November 5. The four new Kepler planets are labeled and plotted as diamonds. Kepler-7 has an unusually low density.

(A color version of this figure is available in the online journal.)

The combined template spectrum for Kepler-7 from FIES was analyzed by A. Sozzetti using MOOG,${ }^{16}$ to provide the stellar parameters needed to estimate the mass and radius of the host star using stellar evolution tracks. The critical input parameters to the models are $T_{\text {eff }}$ and $[\mathrm{Fe} / \mathrm{H}]$, but the spectroscopic $\log g$ is also of interest for a consistency check. A spectrum of Kepler-7 obtained by H. Isaacson and G. Marcy with HIRES on Keck 1 was analyzed by D. Fischer using Spectroscopy Made Easy (SME) (Valenti \& Piskunov 1996), with very similar results: $T_{\text {eff }}=5933 \pm 44$ versus $6000 \pm 75 \mathrm{~K},[\mathrm{Fe} / \mathrm{H}]=+0.11 \pm 0.03$ versus $+0.13 \pm 0.07$, and $\log g=3.98 \pm 0.10$ versus $4.00 \pm$ 0.10 (cgs), for SME and MOOG, respectively. For the results reported in Table 2, we used the SME values. The mean absolute velocity of Kepler-7, $+0.40 \pm 0.10 \mathrm{~km} \mathrm{~s}^{-1}$, was determined from the FIES observations by adopting $-21.508 \mathrm{~km} \mathrm{~s}^{-1}$ as the velocity for the standard star HD 182488.

\section{DISCUSSION}

The analysis of the Kepler photometry and the determination of the stellar and planetary parameters for Kepler-7 followed exactly the procedures reported in Koch et al. (2010) and Borucki et al. (2010). The results are reported in Table 2. These results were checked and confirmed by independent analyses carried out by C. Burke and G. Torres.

The Kepler-7 host star is not much hotter than the Sun, $T_{\text {eff }}=6000 \pm 75 \mathrm{~K}$. However, it is more massive and considerably larger than the Sun, $M_{\star}=1.347_{-0.054}^{+0.072} M_{\odot}$ and $R_{\star}=1.843_{-0.066}^{+0.048} R_{\odot}$, which puts it in a region of the H-R diagram near the end of its main-sequence lifetime. Indeed, the Yale-Yonsei evolutionary tracks (Yi et al. 2001) have hooks that cross at the position of Kepler-7, and the probability distribution for the stellar mass has two peaks. The stronger peak is for an evolutionary state not long before hydrogen burning in the core is exhausted with $M_{\star}=1.362 \pm 0.040 M_{\odot}$ and $R_{\star}=1.857 \pm 0.047 R_{\odot}$, while the weaker peak corresponds to a state soon after the star starts to evolve rapidly, with $M_{\star}=1.204 \pm 0.035 M_{\odot}$ and $R_{\star}=1.781 \pm 0.042 R_{\odot}$. The mass for the evolved peak is $12 \%$ smaller, and the radius

\footnotetext{
16 http://verdi.as.utexas.edu/moog.html
} 
is $4 \%$ smaller (as it must be to yield the same stellar density). The corresponding planetary radius is also $4 \%$ smaller, while the planetary mass is $8 \%$ smaller (because of the dependence on the $2 / 3$ power of the system mass). As our best guess for the mass and radius of the host star and for the mass, radius, and density of the planet, in Table 2 we report the mode and errors for the corresponding probability distributions. This takes into account all the possible evolutionary states for the host star that are consistent with the observations.

The planetary radius is $50 \%$ larger than that of Jupiter, $R_{\mathrm{P}}=1.478_{-0.051}^{+0.050} R_{\mathrm{J}}$, but the mass is less than half, $M_{\mathrm{P}}=$ $0.433_{-0.041}^{+0.040} M_{\mathrm{J}}$, which leads to an unusually low density of $\rho_{\mathrm{P}}=0.166_{-0.020}^{+0.019} \mathrm{~g} \mathrm{~cm}^{-3}$. Among the known planets, only WASP-17b appears to have a lower density (Anderson et al. 2010), although the actual value for that planet is not yet well determined. The position of Kepler-7b on the mass/radius diagram is illustrated in Figure 3, which plots all of the transiting planets with known parameters as of 2009 November 5. Because of possible systematic errors in the radial velocities measured using FIES, the mass of Kepler-7b may be smaller than we report by as much as $20 \%$ or even more. However, the systematic error in the mass on the high side is unlikely to be this large, because a larger orbital amplitude is less vulnerable to systematic velocity errors. For the planetary radius, it is hard to avoid the conclusion that the planet is strongly inflated, because the relatively long duration of the transit demands a low density and expanded radius for the star. A robust measure of the transit duration is the time between the moment when the center of the planet crosses the limb of the star during ingress and the corresponding moment during egress. A general formula for this duration including the effect of orbital eccentricity is given by Pál et al. (2010), leading to a value of $4.63 \pm 0.06 \mathrm{hr}$ for Kepler-7. We conclude that future observational refinements to the characteristics of Kepler-7b are more likely to decrease the density than increase it, with a significant uncertainty remaining as long as the evolutionary state of the host star is uncertain.
Many people have contributed to the success of the $\mathrm{Ke}$ pler Mission, and it is impossible to acknowledge them all by name. We offer our special thanks to the team of scientists and programmers working with J. M. Jenkins to create the photometric pipeline-H. Chandrasekaran, S. T. Bryson, J. Twicken, E Quintana, B. Clarke, C. Allen, J. Li, P. Tenenbaum, and $\mathrm{H}$. Wu; to C. J. Burke and G. Torres for running independent checks of the analysis of the Kepler-7 light curve and system parameters; to J. Andersen for help with the FIES observations and unwavering moral support; to M. Endl, H. Isaacson, D. Ciardi, G. Mandushev, N. Baliber, and M. Crane for important contributions to the follow-up work; to A. Sozzetti for his analysis of the FIES combined template spectrum and to D. Fischer for her analysis of the HIRES template spectrum; to M. Everett and G. Esquerdo for critical contributions to the KIC; to E. Bachtel and his team at Ball Aerospace for their work on the Kepler photometer; to R. Duren and R. Thompson for key contributions to engineering; and to C. Botosh, M. Haas, and J. Fanson, for able management. D. W. L. gratefully acknowledges partial support from NASA Cooperative Agreement NCC2-1390 and the help of S. Cahill and L. McArthur-Hines. Funding for this Discovery mission is provided by NASA's Science Mission Directorate.

$\begin{array}{ll}\text { Facilities: Kepler, NOT(FIES), Keck:I(HIRES), } & \end{array}$

\section{REFERENCES}

Anderson, D. R., et al. 2010, ApJ, 709, 159

Batalha, N. M., et al. 2010, ApJ, 703, L103

Borucki, W. J., et al. 2010, ApJ, 703, L126

Gautier, T. N., et al. 2010, ApJ, submitted

Koch, D. G., et al. 2010, ApJ, 713, L131

Kurucz, R. L. 1992, in IAU Symp. 149, The Stellar Populations of Galaxies, ed. B. Barbuy \& A. Renzini (Dordrecht: Kluwer), 225

Monet, D. G., et al. 2010, arXiv:1001.0305

Pál, A., et al. 2010, MNRAS, 401, 2665

Valenti, J. A., \& Piskunov, N. 1996, A\&AS, 118, 595

Yi, S. K., Demarque, P., Kim, Y.-C., Lee, Y.-W., Ree, C. H., Lejeune, T., \& Barnes, S. 2001, ApJS, 136, 417 